\title{
Special issue on recent advances in control of discrete event systems
}

\author{
Antonio Ramírez-Treviño • Manuel Silva • \\ Stéphane Lafortune
}

Published online: 22 February 2015

(C) 2015 Springer Science+Business Media New York

This special issue of J-DEDS on Recent Advances in Control of Discrete Event Systems contains 13 papers that cover a wide range of recent advances on the modeling, analysis, control, and optimization of discrete event systems, for untimed, timed, and stochastic models. These submissions were carefully reviewed, following the standard editorial process for full papers in J-DEDS. Finally, all 13 papers were selected for this special issue. We note that a complementary special section dealing with fluid and hybrid approximations of discrete event systems modeled by Petri nets, appears in the journal Nonlinear Analysis: Hybrid Systems, volume 12, 2014.

A brief description of each paper in this special issue follows.

In "Multi-intersection Traffic Light Control with blocking", Yanfeng Geng and Christos G. Cassandras develop a stochastic flow model for a set of traffic intersections in vehicular networks. Using Infinitesimal Perturbation Analysis techniques, they obtain estimators that are used to determine optimal cycle lengths of the traffic lights. DOI: 10.1007/S10626-013-0176-0

In "Maximally permissive deadlock avoidance for resource allocation systems with R/Wlocks", Ahmad Nazeem and Spyros Reveliotis consider liveness-enforcing supervision of a type of resource allocation systems called "readers-writer". They exploit special features of the system's space to obtain maximally permissive solutions. DOI: 10.1007/S10626-014-0202-X

In "Coordination control of discrete-event systems revisited", Jan Komenda and colleagues revise and improve the coordination in supervisory control of distributed discrete event systems with synchronous communication. Conditional decomposability, controllability, and closedness concepts are carefully revised and generalized; moreover, optimization of the size of the coordinator is considered. DOI: 10.1007/S10626-013-0179-X

In "A hierarchical and modular control architecture for sequential behaviours", Christine Baier and Thomas Moor develop a hierarchical and modular control architecture for plant

A. Ramírez-Treviño $(\bowtie)$

Cinvestav, Guadalajara, México

e-mail: art@gdl.cinvestav.mx

M. Silva

Universidad de Zaragoza, Zaragoza, Spain

e-mail: silva@unizar.es

S. Lafortune

The University of Michigan, Ann Arbor, MI, USA

e-mail: stephane@umich.edu 
dynamics and specifications represented as formal languages. Based on some structural properties, they propose to modularly (i.e., bottom-up) construct a hierarchical control system by alternating controller design, closed-loop composition, and abstraction. DOI: 10.1007/ S10626-014-0182-X

In "Computation of supervisors for reconfigurable machine tools", Klaus W. Schmidt addresses the problem of efficient reconfiguration of machine tools in flexible manufacturing systems. A polynomial-time algorithm for the construction of a reconfiguration supervisor is presented; the modular realization of this supervisor enables controller modifications such as adding or removing configurations during run-time. DOI: $10.1007 /$ S10626-014-0183-9

In "Robustness of synchronous communication protocols with delay for decentralized discrete-event control", Waselul H. Sadid and colleagues investigate the effect of delay in decentralized control architectures with communication. Specifically, they investigate the robustness with respect to non-zero but finite delay of synchronous communication protocols where not all observations are communicated. DOI: 10.1007/S10626-014-0184-8

In "Hierarchical modelling of manufacturing systems using discrete event systems and the conflict preorder", Robi Malik and Ryan Leduc further investigate the technique of Hierarchical Interface-Based Supervisory Control for the design of complex systems. Using the notion of conflict preorder, they provide increased flexibility for the interface consistency conditions over previous approaches. DOI: 10.1007/S10626-014-0185-7

In "New results on supervisor localization, with case studies", Kai Cai and W.M. Wonham further develop the technique of supervisor localization, a top-down approach to distributed control of discrete event systems. They first present several refinements to localization theory and then apply their results to two case studies: multi-agent formation and cluster-tool control. DOI: 10.1007/S10626-014-0194-6

In "Employing behavioral preorders to define controllability for nondeterministic discreteevent systems", Jasen Markovski uses a partial bisimulation preorder, thus a process-theoretic perspective, to deal with the notion of controllability for nondeterministic discrete event systems. The induced equivalence relation is employed to minimize the original model of the unsupervised system, providing an efficient algorithm. DOI: 10.1007/S10626-014-0201-Y

In "Enforcing opacity of regular predicates on modal transition systems", Philippe Darondeau and colleagues lift the problem of opacity enforcement by supervisory control from finite transition systems to families of finite transition systems specified by modal transition systems. The goal is to enforce the opacity of a secret predicate on all models of a modal transition system. (Tragically, Philippe Darondeau died during the preparation of this special issue. We mourn the loss of a distinguished researcher and warm friend.) DOI: 10. 1007/S10626-014-0193-7.

In "Diagnosis and opacity problems for infinite state systems modeled by recursive tile systems", Sébastien Chédor and colleagues study opacity and diagnosability problems in the setting of recursive tile systems (RTSs), a class of infinite-state systems. The complexity class of each decidable result is established. DOI: 10.1007/S10626-014-0197-3

In "New representations for $(\max ,+)$ automata with applications to performance evaluation and control of discrete event systems", Rabah Boukra and colleagues deal with automata with weights in the so-called $(\max ,+)$ algebra. Specific recursive equations help to describe their extremal behaviors. Both performance evaluation and control (i.e., exogenous influences) issues are considered. DOI: 10.1007/S10626-013-0178-Y

Finally, in "Compositions of ( $\max ,+$ ) automata", Sébastien Lahaye and colleagues propose two types of synchronous product operations for $(\max ,+)$ automata, a class of models for safe timed Petri nets, thereby allowing composition of sub-models when modeling a complex 
system. In addition, an asynchronous product is also proposed for a class of bounded timed Petri nets. DOI: 10.1007/S10626-014-0186-6

We are grateful to the authors for their contributions and timely revisions. We also thank the reviewers for their careful reading and their numerous helpful comments and suggestions. Finally, we thank past Editor-in-Chief of J-DEDS, Xi-Ren Cao, for inviting us to serve as guest editors for this special issue.

Antonio Ramírez-Treviño, Manuel Silva, Stéphane Lafortune Guest Editors

Antonio Ramírez-Treviño received the Bachelor Engineering degree in Electrical Engineering from the Universidad Autónoma Metropolitana, Mexico D. F. in 1986, the Master of Science degree in Automatic Control from the Cinvestav Zacatenco, Mexico D. F. in 1990, and the Ph. D. degree in Systems Engineering from the Universidad de Zaragoza in 1993, Zaragoza, Spain. His current research interests include Discrete Event ans Hybrid Systems, focusing in problems related with the controllability, observability and diagnosability of these systems as well as applying the derived results to the industrial problems. Currently, he is with the Centro de Investigación y de Estudios Avanzados del IPN at Guadalajara, Mexico as a professor of the Electrical Engineering Department.

Manuel Silva received the Industrial-Chemical Engineering degree from the University of Sevilla, Sevilla, Spain, in 1974, and the postgraduate and Ph.D. degrees in control engineering from the Institut National Polytechnique de Grenoble, Grenoble, France, in 1975 and 1978, respectively. In 1978, he started the group of Systems Engineering and Computer Science at the University of Zaragoza, Zaragoza, Spain, where he was appointed Professor, in 1982. At present, his research interests include modeling, validation, performance evaluation, and implementation of large concurrent systems using Petri nets. He is the author of the book Las Redes de Petri en la Automática y la Informática (AC, 1985; reprinted: Thomson, 2003), coauthor of Practice of Petri Nets in Manufacturing (Chapman \& Hall, 1993), and co-editor of an special issue on CIM of the IEEE TRANSACTIONS ON ROBOTICS AND AUTOMATION. Dr. Silva was Dean of the Centro Politécnico Superior, University of Zaragoza, from 1986 to 1992, and President of the Aragonese Research Council (CONAI) and of the Research and Innovation Committee of the French-Spanish Comisión de Trabajo de los Pirineos, from 1993 to 1995 . He has been Associate Editor of the IEEE TRANSACTIONS ON ROBOTICS AND AUTOMATION, of the European Journal of Control and of the Journal of Discrete Event Systems. Associate editor of the Trans. on Petri Nets and Other Models of Concurrency, is advisory member of the IEICE Trans. on Fundamentals on Electronics, Communications, and Computer Sciences and of the Journal of Discrete Event Systems. Member of the Steering Committees of the Int. Conf. on Application and Theory of Petri Nets and IFAC Conf on Analysis and Design of Hybrid Systems, also in the past for the Int. Workshops on Discrete Event Systems. Founder member of the Asociación Española de Robótica, interested in the history of technology, he is the editor of the series of books on the history of Técnica e Ingeniería en España (7 vols. edited). Distinguished with a medal from Lille, by the Association of Telecommunication Engineers of Aragón, and Doctor Honoris Causa by the Universite de Reims-Champagne-Ardennes, he is a member of the Royal Academy of Engineering of Spain.

Stéphane Lafortune (M'82-SM'97-F'99) received the B.Eng. degree from Ecole Polytechnique de Montréal, Montreal, QC, Canada, in 1980, the M.Eng. degree from McGill University, Montreal, in 1982, and the Ph.D. degree from the University of California at Berkeley in 1986, all in electrical engineering. Since September 1986, he has been with the University of Michigan, Ann Arbor, where he is a Professor of Electrical Engineering and Computer Science. Dr. Lafortune is a Fellow of the IEEE (1999). He received the Presidential Young Investigator Award from the National Science Foundation in 1990 and the George S. Axelby Outstanding Paper Award from the Control Systems Society of the IEEE in 1994 (for a paper co-authored with S. L. Chung and F. Lin) and in 2001 (for a paper co-authored with G. Barrett). Dr. Lafortune's research interests are in discrete event systems and include multiple problem domains: modeling, diagnosis, control, optimization, and applications to computer systems. He is the lead developer of the software package UMDES and co-developer of DESUMA with L. Ricker. He co-authored, with C. Cassandras, the textbook Introduction to Discrete Event Systems-Second Edition (Springer, 2008). Dr. Lafortune is a member of the editorial boards of the Journal of Discrete Event Dynamic Systems: Theory and Applications and of the International Journal of Control. 